Revista Posgrado y Sociedad

Sistema de Estudios de Posgrado

Universidad Estatal a Distancia

ISSN 2215-2172

Costa Rica

revistaposgradoysoci@uned.ac.cr

\title{
La investigación en la formación de educadores: algunas estrategias para la Escuela de Ciencias de la Educación de la UNED Costa Rica
}

\author{
Teacher Educators as researchers: Knowledge, Management and \\ Development \\ Jensy Campos Céspedes \\ Universidad Estatal a Distancia \\ San José, Costa Rica \\ Volumen 12, Número 1 \\ Marzo 2012 \\ pp. 1 - 21
}

Recibido: Marzo,2011

Aprobado: Agosto, 2011 


\begin{abstract}
Resumen
En este documento se presentan algunas reflexiones en torno a la importancia de la investigación en las carreras de Educación y tiene el propósito de recordar la necesidad de fortalecer el quehacer investigativo y la articulación de las funciones sustantivas de la universidad en los procesos académicos desarrollados por las unidades formadoras de docentes, a fin de propiciar un quehacer asentado en la investigación orientada al desarrollo. De acuerdo con las características actuales, la investigación aplicada a la práctica docente y a los fenómenos que afectan los procesos educativos han de ser los principales insumos requeridos para la gestión del conocimiento y la formación de profesionales en Educación, capaces de aprender a aprender y con competencias para gestionar conocimiento. Ello supone una resignificación de la educación en los procesos de construcción de un nuevo imaginario de sociedad caracterizado por la inclusión y el desarrollo. En este sentido, se reconoce que la gestión del conocimiento no solo impacta en el desarrollo del currículo, sino que tiene resonancia en la construcción y reconstrucción de la educación y del educador.
\end{abstract}

Palabras claves: Gestión del conocimiento, formador de formadores, investigación educativa.

\begin{abstract}
This article presents some reflections on the need of universities in Costa Rica to raise the quality of research that occurs in careers on education, to foster a task based on research oriented toward development.

Applied research in educational practice and the phenomena that affect the educational process must be the main inputs required for knowledge management and training of professionals capable of learning to learn skills and knowledge to manage. This implies a re-education in the process of building a new imaginary of society characterized by the inclusion and development. In this sense, it is recognized that knowledge management not only impacts the development of curriculum, but it has resonance in the construction and reconstruction of education and educators, from the ontological, political and axiological dimensions.
\end{abstract}

Keywords: Knowledge management, reeducating teacher educators, educational research. 


\section{Introducción}

"En última instancia, la educación superior debería apuntar a crear una nueva sociedad no violenta y de la que esté excluida la explotación, sociedad formada por personas muy cultas, motivadas e integradas, movidas por el amor hacia la humanidad y guiadas por la sabiduría."(Tunnerman y Souza, 2003, p.11)

El tema del docente como investigador ha sido objeto de múltiples disertaciones asociadas con la calidad educativa, con la profesionalización docente y con la necesidad de reposicionar la Educación como actividad base de la transformación (Cañal, Lledo, Pozuelo y Trave, 1997). En el contexto costarricense, la investigación en Educación constituye un asunto crítico pues los cambios políticos, sociales, económicos y culturales exigen a la Educación fundamentar sus saberes en la gestión del conocimiento en razón de que surgen nuevas demandas sociales para la educación, emergen nuevos escenarios educativos y se reconfiguran las prácticas pedagógicas y los sujetos epistémicos. Esas modificaciones plantean nuevas demandas y desafíos a los formadores de educadores (Campos y Chinchilla, 2009).

La complejidad actual permea todos los espacios - públicos y privados- y coloca a los profesionales de la educación en una situación difícil al tener ante sí una población estudiantil con condiciones socioeconómicas, emocionales y hasta cognitivas, diferentes a las que hace algunas décadas tenían otras generaciones.

El desarrollo de las Tecnologías de la Información y la Comunicación (TIC) y la globalización han discurrido en significativos distanciamientos entre generaciones; una brecha digital, formas de interacción, comprensión y reelaboración de la realidad distintas (Campos y Chinchilla, 2009). La población estudiantil presente en los escenarios educativos no es la misma para la cual, en la mayoría de los casos, se diseñó el sistema educativo tradicional (Prensky, 2001; Santoianni \& Striano, 2006).

Este hecho, aunado a que uno de los principales criterios de calidad educativa está dado por la pertinencia social (UNESCO, 1998), antepone a las carreras de Educación la necesidad de constituirse en gestoras de conocimiento. El concepto de gestión se entiende en su sentido más complejo, que incluye el proceso sistemático de producción, recuperación, custodia, uso y reelaboración del conocimiento.

En consecuencia de lo anterior, las competencias de los formadores de docentes actualmente exigen que sus procesos de aprendizaje estén basados en la producción de conocimientos desarrollados a partir del quehacer académico-investigativo de los formadores. Tal como lo indica Imbernón (2002): "investigar en educación es necesario para generar cambios, para revisar el conocimiento educativo constituido por la evidencia, la experimentación y la intuición para generar nuevos conocimientos que permitan generar una mejor educación de los ciudadanos" (p. 7). Asimismo, las demandas hacia la universidad exigen que se trascienda el quehacer universitario centrado en la formación 
de profesionales y se asuma la responsabilidad socialmente conferida de gestionar conocimiento útil, para orientar al país por los derroteros del desarrollo social e individual de todas las personas que lo habitan (Goulet, 1965).

\section{Universidad y gestión del conocimiento}

Reflexionar respecto de la investigación por parte de los formadores de profesionales de la Educación pasa por la puesta en la palestra de algunas interrogantes: ¿cuál es la esencia del ser universidad y de la academia?, ¿cuáles son las bases fundantes de la educación y la formación de educadores?, ¿cuáles son los elementos claves que garantizan pertinencia social en contextos complejos tendientes a la segmentación social?

Sin pretender dar respuestas simplistas a estas interrogantes $y$, menos aún, arrogarnos verdades absolutas, se presentarán algunas ideas generales para orientar la reflexión, de manera que esta conlleve a la puesta en marcha de acciones que permitan el mejoramiento de la formación de formadores y el quehacer educativo, en una Costa Rica donde cada vez más parecen desdibujarse algunos principios fundantes de la sociedad democrática, solidaria y equitativa a la que en algún momento se aspiró.

Uno de los fenómenos más importantes de la nueva forma de configuración de sociedad ha sido la globalización que se ha precipitado por la explosión del desarrollo de las TIC. La globalización es un fenómeno multidimensional, que afecta todas las esferas públicas y privadas, y da lugar a cambios sustanciales no solo socioeconómicos, sino también culturales y psicológicos que hacen que las relaciones e intersubjetividades estén en construcción permanente.

El desarrollo tecnológico y el proceso de globalización han impulsado una resignificación del conocimiento y lo ubican como el factor de producción más importante, en especial por el aumento de la incertidumbre y el surgimiento de nuevos y complejos escenarios. En este sentido, autores como Melucci (1998) señalan la proliferación de espacios públicos, la politización de la esfera privada, el surgimiento de la ciudadanía como forma de identificación, la producción y circulación acelerada de bienes simbólicos y la complejización de la acción social (Melucci, 1998; Moffe, 1993 citados por Fuentes, 2001). En este contexto, las universidades deben asumir funciones, estrategias e interacciones con otros agentes sociales, a fin de asegurar que el conocimiento sea gestionado y utilizado para sustentar procesos de transformación y desarrollo social. Ello implica establecer mecanismos para crear y transferir conocimientos dentro de una estrategia articulada a las funciones académicas de la institución (Pérez, 1998).

La vinculación de la universidad con el conocimiento, la formación de 
profesionales y la labor de extensión e investigación son acciones que deben someterse a reflexión en los nuevos escenarios universitarios, pues si bien son las funciones académicas sustantivas de la universidad, también es cierto que no todas las universidades logran desarrollar y articularlas adecuadamente para lograr que la producción del conocimiento influya en el desarrollo de la sociedad. En otras palabras, para las unidades académicas de la UNED es vital incluir en los procesos de autoevaluación la revisión de su quehacer a la luz de lo que múltiples autores (Brunner et al 2005, Casas, 2005; Díaz, 2008; Didrikison, 2007; Rama, 2008) denominan la responsabilidad socialmente conferida respecto de la producción, uso y democratización del conocimiento. Ello supondría analizar el concepto conocimiento y su importancia como sustento fundamental de la vida en una sociedad en la que "ya no se caracteriza por producir cosas, sino conocimiento..." (De Miguel, 2003, p. 15) y cumplir con los propósitos establecidos por el Plan Nacional de Educación Superior 2006-2010 referidos a apoyar la definición de políticas públicas en educación, desarrollar núcleos de investigación científica y tecnológica y fortalecer el vínculo con las comunidades (CONARE, 2005, p.27).

En el análisis de esos desafíos y desde una mirada evaluativa al quehacer de las unidades académicas formadoras de docentes, tal como se desprende del segundo informe del Estado de la Educación del país, hay una brecha importante que cerrar entre lo que las universidades logran realizar y lo que la sociedad está requiriendo que se haga urgentemente (Estado de la Educación en Costa Rica, 2008).

El fortalecimiento de la investigación en educación y la gestión del conocimiento por parte de las unidades académicas universitarias del campo de la Educación, se derivan necesidades concretas como la formación de competencias para la investigación, concebida como la capacidad de pensar y actuar con creatividad y perseverancia, de manera individual o colectiva para interpretar hechos o fenómenos, como una forma de construir nuevos conocimientos y aplicarlos en la resolución de problemas. Lo anterior debe incluir la gestión adecuada de procedimientos con una apertura hacia lo nuevo y lo inesperado (Fromm y Ramón, 2002). Dicho de otra manera, se requiere una capacidad de problematización y recreación de la realidad a partir de la investigación. Dicha competencia debe desarrollarse en los formadores titulados por las universidades $y$, necesariamente, en el personal docente que tiene bajo su responsabilidad formar profesionales de la Educación.

Corresponde a los formadores de formadores gestionar conocimiento en los campos de saber que confluyen en la educación y reclamar un lugar en los espacios sociales y políticos que asignan valores a las profesiones. Esta presencia se logra con conocimientos y propuestas debidamente fundamentadas. Esto es difícil de lograr sin investigar.

En esa línea, Imbernón (2007) indica que sin investigar no hay campo de conocimiento; hay rutina, copia, reproducción dependencia y estatismo. 
"La educación y la enseñanza como prácticas sociales imprescindibles para el progreso de la humanidad, requieren por un lado, un proceso de investigación constante y, por otro lado, que el conocimiento generado sea analizado y compartido mediante la formación de sus protagonistas activos" (p.7).

A la luz de lo anterior, conviene señalar dos asuntos críticos en la resignificación de la educación: la revaloración del formador como profesional y como científico de la educación y su posicionamiento político.

Por otra parte, la incorporación de la investigación como reflexión en la acción no solo conlleva a una reconsideración del educador como profesional, sino que, en consecuencia, conduce a una reconceptualización teórico-práctica de su formación (Muñoz, 2001). Esto, ineludiblemente, transversaliza la práctica docente, los fundamentos filosóficos y pedagógicos que sustentan la formación de los formadores de acuerdo con las nuevas corrientes de pensamiento y las nuevas condiciones sociales políticas y económicas que envuelven el proceso formativo en las instituciones de educación superior.

La Declaración mundial sobre educación para todos y la Propuesta para la educación XXI señalan la necesidad de generar propuestas educativas innovadoras que fomenten hábitos, competencias y destrezas para la indagación, reflexión y análisis crítico (From y Ramón, 2002). Estas, a su vez, son clave para que los educadores garanticen el aprendizaje a lo largo de la vida (Delors, 1996).

La incorporación de la investigación como elemento clave $\mathrm{y}$ fundante de la formación de profesionales de la educación conlleva una ruptura que se explica por el reclamo de nuevas teorías (pedagógicas, sociológicas, antropológicas, psicológicas y neurológicas) y su incorporación en la construcción del discurso y la práctica educativa. Esto conduce a dos conceptos clave: la interdisciplinaridad y la transdisciplinaridad. Y sobre todo, a comprender que el conocimiento tiene caducidad (Pozo, 2010) y ello significa que el debate debe orientarse a determinar nuestras concepciones epistemológicas.

Atender pertinentemente las demandas sociales en escenarios sociales altamente complejos es difícil de lograr desde la disciplinariedad. Formar el educador requerido exigirá la incorporación de saberes de otras disciplinas o campos del saber. Esto supone, a las carreras de Educación, reflexionar acerca de las características que su cuerpo académico requiere, frente a los cambios en el perfil de profesionales de la educación que se desean formar.

De acuerdo con los planteamientos de investigadores del quehacer universitario se puede observar que la identificación de líneas de investigación, la articulación en los procesos académicos de la investigación, la docencia y la extensión 
en los que participen docentes y estudiantes -como pueden ser los Núcleos Integradores de Docencia Investigación y Acción Social, semilleros de investigación- se constituyen en escenarios deseables para la concreción de una praxis pedagógica-investigativa desde una carrera de educación.

Figura 1

Modelo para la gestión del conocimiento en la Escuela de Ciencias de la Educación

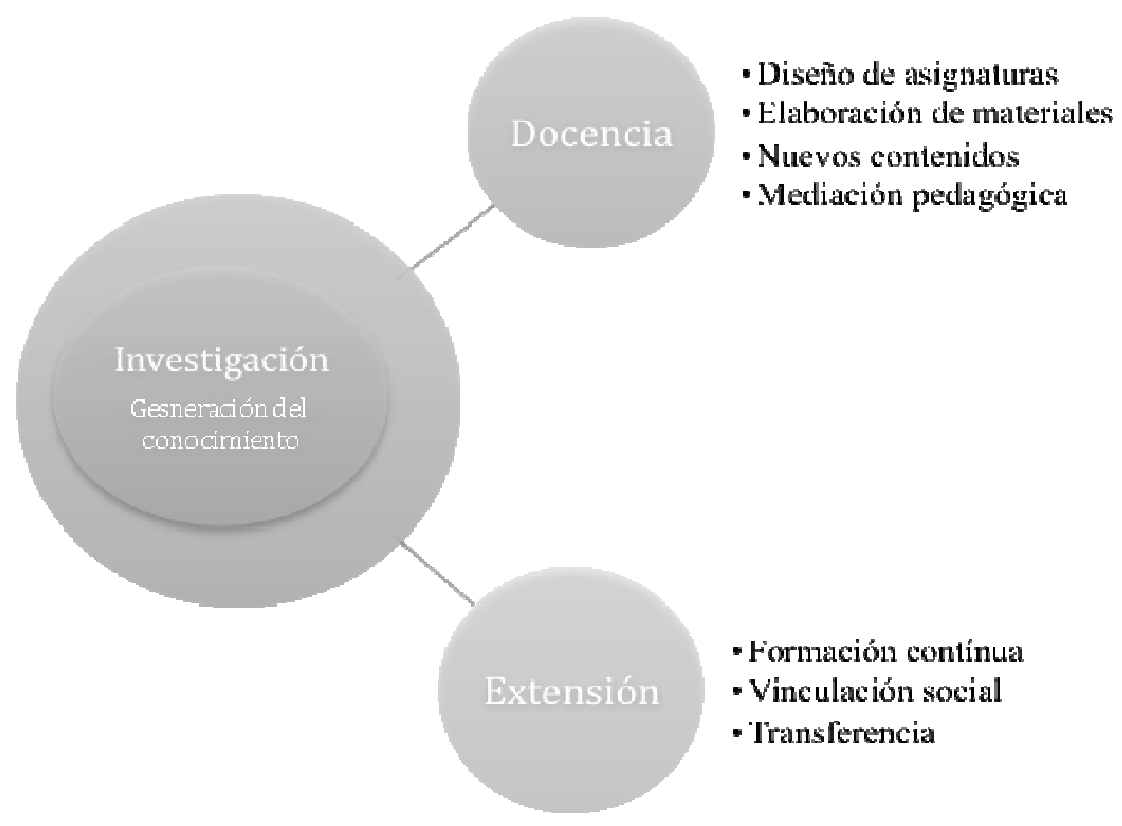

Fuente: Elaboración propia, 2011. 
El concepto de universidad analizado permite rescatar las funciones fundamentales de la Escuela de Ciencias de la Educación (ECE) como instancia académica. Con esto se resignifica su personal académico, pues se reconoce en él la función de investigador como parte de su esencia. Asimismo, se evidencia la responsabilidad de las instancias de apoyo de colaborar para que los académicos logren cumplir responsablemente sus funciones. Cercenar del personal académico la labor investigativa conduce, no solo a que este personal no cumpla la función socialmente conferida de generar conocimiento, sino que, consecuentemente, los procesos formativos y de extensión se ven limitados por el vacío de investigación.

La investigación para apoyar los procesos académicos puede ser reforzada por otras instancias universitarias, sin embargo, en primera instancia, la responsabilidad de la investigación es del personal académico de la institución universitaria.

Otro asunto crítico por atender para elevar la producción investigativa en las carreras de Educación de la ECE, se refiere a la necesidad de identificar líneas de investigación a partir de los vacíos de conocimiento existentes, y en aras de resolver o mejorar las respuestas a los problemas relacionados con la educación. Cabe indicar que ya en el país existen importantes insumos que podrían ayudar a establecer líneas de investigación para las carreras de educación. Sin embargo, es claro que la reflexión y el proceso de consulta a los actores sociales involucrados con el fenómeno educativo son indispensables para constituir una agenda de investigación en educación que permita la ampliación, profundización y la articulación de esfuerzos investigativos, tanto intrainstitucional como interinstitucionalmente.

La resignificación del quehacer docente en la ECE y el desplazamiento de la investigación hacia el centro de la vida académica exige la reconsideración de la estructura de asignación y gestión de recursos y una cuidadosa evaluación de otros aspectos administrativos, como por ejemplo:

-las funciones asignadas al personal académico, en especial de los puestos de coordinación de cátedra o de programa.

-los requisitos, mecanismos de selección y contratación. En este sentido, es importante revalorar los perfiles que la institución establece para los tutores y para los coordinadores de cátedra y programas, en función de los desarrollos que se espera alcanzar para la ECE en términos de investigación, proyección del aprendizaje en línea y de la internacionalización de la oferta académica.

-los procesos de formación continua y los espacios de desarrollo profesional que se están ejecutando y su efecto en los procesos académicos.

Al seguir el planteamiento anterior, también se hace necesario operacionalizar las políticas y 
normativas institucionales que ubican a la investigación como elemento clave en el quehacer universitario (Morales, 2011) y renunciar a ser parte de las "varias universidades (que) poseen adecuadas políticas sobre la estructura y el desarrollo de la investigación; sin embargo, no existe planta de docentes investigadores, no poseen infraestructura para la investigación, por lo tanto, no existen líneas de investigación” (Torres, 2005, p. 3).

\section{La formación para la investigación en carreras de Educación}

La formación para la investigación en los estudiantes de las carreras de Educación, o para cualquier campo del saber, no se resuelve con la prescripción de metodologías de investigación, sino con la vivencia de la investigación como parte del proceso formativo. Según Gimeno Sacristán y Pérez Gómez, 1983; Goetz y LeCompte, 1988 (citados por Imbernón, 2002), la investigación, como estrategia de enseñanza aprendizaje, es altamente potente en la formación de los educadores.

La formación para la investigación en las carreras de la ECE tradicionalmente se ha caracterizado por la existencia de uno o dos cursos de investigación. Para la mayoría de las carreras de la ECE en su plan de estudios solamente se cuenta con un curso de investigación sobre métodos y técnicas de investigación (Campos y Castro, 2010). Asimismo, pese a que existen ejercicios investigativos o la elaboración de actividades académicas que conllevan la aplicación de algunas técnicas de investigación, estos ejercicios no están organizados ni obedecen a una decidida, organizada y sistemática transversalización de la investigación dentro de los planes de estudio de las carreras (Campos e Hidalgo, 2011).

La formación para la investigación en la ECE se ha caracterizado por coexistir con condicionantes que limitan la creación de una cultura de investigación y que riñen con el concepto de docente investigador que debería desarrollar el cuerpo académico de la Escuela y los profesionales formados por la misma. El análisis de los contenidos y del material de estudio que tradicionalmente se ha utilizado, al menos hasta hace tres años, el curso de investigación que se ofrece a todas las carreras de educación permite observar la presencia del paradigma positivista con posición hegemónica frente a otros paradigmas investigativos, una baja producción investigativa por parte del personal docente a cargo de los cursos de investigación

fundamentalmente brindan sus servicios un cuarto de tiempo para la universidad y de forma residual a su ocupación laboral principal. Asimismo, esos condicionantes encuentran contraparte en una población estudiantil que manifiesta expresiones asociadas con prejuicios y mitos que socialmente se han configurado en torno a la investigación y que la conciben como una actividad difícil, poco ligada con la cotidianidad de las personas y, en consecuencia, con baja utilidad. Así como esos condicionantes negativos que se encuentran en el 
interior de la cátedra, existen, además, otros condicionantes a nivel de la ECE, y a nivel de la UNED en general, que dificultan la formación para la investigación (observar figura 2). En el nivel macro, en la ECE sobresale una ausencia de cultura investigativa, la desarticulación de los escasos esfuerzos investigativos, la ausencia de líneas de investigación $\mathrm{y}$, en especial, la delegación de la formación para la investigación a la cátedra de investigación educativa confinando la formación de competencias investigativas a los alcances que los pocos cursos de investigación logran concretar. Esto es el efecto de la ausencia de una planificada y estratégica trasversalización de la investigación en los planes de estudio de las carreras de la ECE y una pobre asignación de espacio a la investigación dentro de las mallas curriculares. Estudios realizados (Campos y Castro, 2010) muestran que las carreras de educación de la UNED presentan menos del $50 \%$ de cursos y de créditos en investigación, en comparación con el resto de universidades públicas del país

\section{Figura 2}

Condicionantes negativos de la formación para la investigación en la cátedra, en la ECE y en la UNED

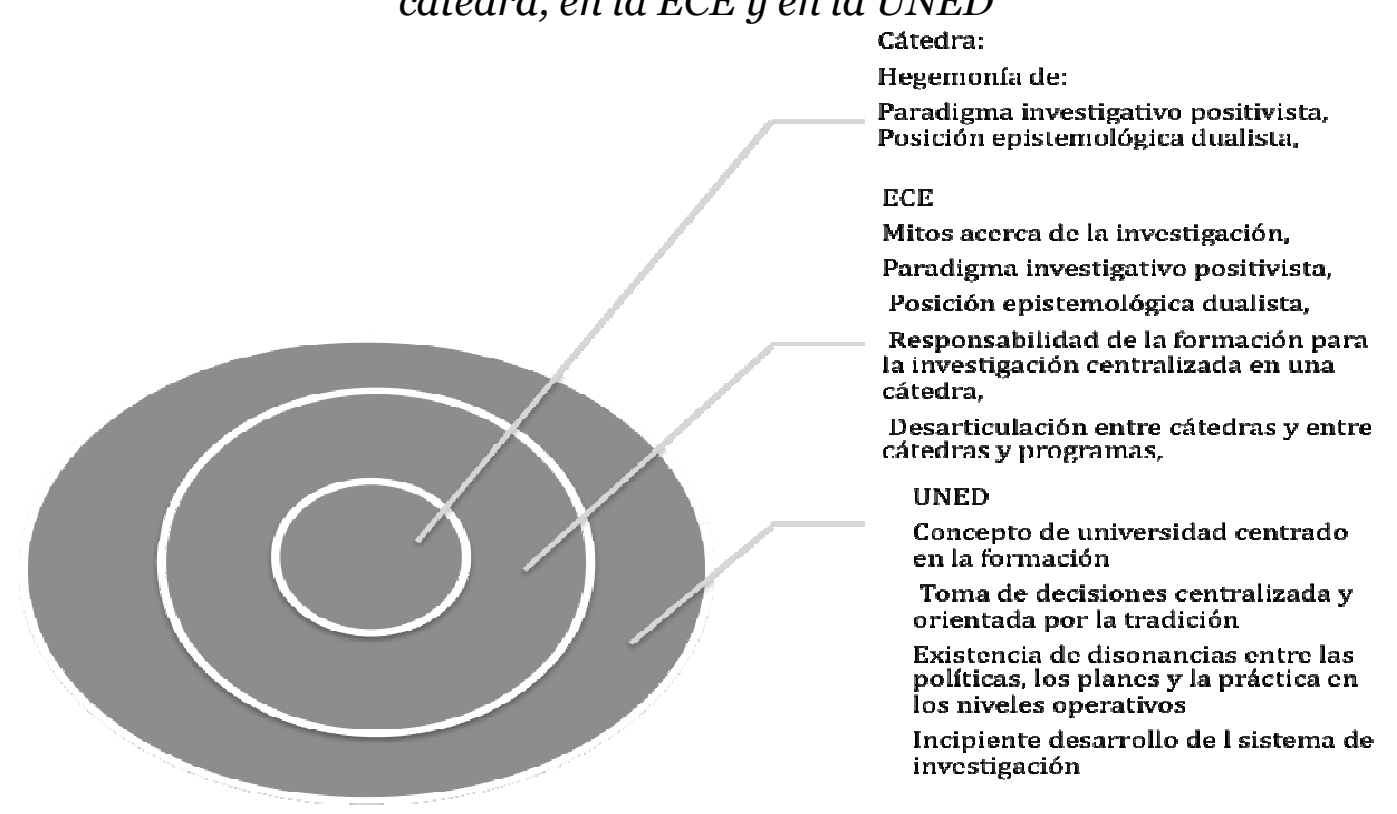

Fuente: Elaboración propia a partir de análisis de sistema realizado a la Cátedra de Investigación Educativa, 2 
Es posible, en definitiva, hacer investigación en Educación, y es posible que los educadores investiguen. En ese sentido, la investigación de aula, la investigación participativa y la investigación acción son metodologías que ofrecen amplitud de posibilidades para formar a los docentes en esta habilidad y, con ello, profundizar en el conocimiento y la comprensión de los fenómenos que se gestan y desarrollan en los contextos educativos, de modo que, a partir de esa comprensión, se generen propuestas pedagógicas socialmente pertinentes (From y Ramon, 2002). En relación con lo anterior, es importante retomar los aportes de Muñoz (2001), quien plantea que la investigación es una indagación sistemática y autocrítica que contribuye a perfeccionar la enseñanza, siempre que el mismo docente compruebe sus hipótesis sin tener que aceptar acríticamente las propuestas de otros (Muñoz, Quintero, Ancízar 2001, p.23).

Por otra parte, pensar en la investigación por parte del formador de docentes conlleva el planteamiento de interrogantes asociadas con su uso, por ejemplo, ¿cómo se logrará que el conocimiento que se produce o se gestiona mediante la investigación llegue a la sociedad? La respuesta depende de las características específicas de la institución y de su modelo de gestión del conocimiento. Pero, en todo caso, es claro que las instituciones formadoras responden a esta interrogante de forma ineludible: la incorporación de los resultados de la investigación que se realiza, en los programas de las asignaturas incluidas en el plan de estudios. Es de esperar que una institución formadora de profesionales de la educación, al menos, desarrolle investigación y establezca mecanismos que viabilicen la inclusión de los productos de las investigaciones como material de estudio de los profesionales en formación. Aunado a lo anterior, en nivel nacional se ha detectado la necesidad de incrementar la producción investigativa en el sector educativo; asimismo, hay consenso en que los profesionales en Educación deben fortalecer las competencias investigativas como una de las formas de inducir el cambio en un sistema educativo nacional, que parece poco acorde con las necesidades y demandas sociales (Alfaro y Francis, 2009; Campos y Chinchilla, 2009; Venegas, 2008).

Según Vessuri (2009), la formación de investigadores requiere reformas para lograr científicos socialmente responsables cuyo trabajo se oriente a la democratización del conocimiento y del asesoramiento experto, ya que los "qué" y los "cómo" del conocimiento asumen valores primordiales y deben estar al servicio de la ciudadanía (pp. 69-70). En consecuencia, la investigación en Educación y los docentes investigadores que constituyen la planta docente de las carreras de Educación han de reconocer en la investigación valores que van más allá, como indica Vessuri, (2009), de la producción de conocimientos y que apunta hacia el mejoramiento de las condiciones de las mayorías y a salvar el medio ambiente.

\section{El uso de las tecnologías de la información y la comunicación en la formación de formadores}

Por otra parte, las carreras de educación deberán desarrollar un plan de incorporación de las TIC en los procesos de enseñanza-aprendizaje y en otros espacios del quehacer académico. 
La utilización de las TIC en los procesos de investigación y de gestión curricular puede potenciar las labores académicas que en este momento requieren fortalecerse. Entonces, se trata de que los conceptos de trabajo colaborativo, aprendizaje social y ruptura de barreras de tiempo y espacio se incorporen a la vida académica de las carreras de educación para enriquecer no solo los procesos formativos de los estudiantes, sino de los formadores también.

En cuanto a la incorporación de las TIC en los cursos, según el Programa de Aprendizaje en línea (2010) en la Escuela de Ciencias de la Educación (ECE) la cantidad de asignaturas que incursiona en la educación en línea ha crecido progresivamente. Sin embargo, la herramienta que más se utiliza es el foro y las actividades académicas que se desarrollan en estos cursos continúan siendo ejercicios individuales (Campos e Hidalgo, 2011). El trabajo colaborativo por parte de los estudiantes es posible en la ECE utilizando herramientas como wikis, software para crear redes sociales, blogs que están disponibles en Internet. Incluso, con la misma herramienta de foro, se puede lograr que los estudiantes trabajen colaborativamente y que haya procesos de aprendizaje social entre los estudiantes de la ECE que geográficamente están dispersos. Para ello, los encargados de cátedra requieren de procesos de asesoramiento y acompañamiento al momento de plantear los diseños de los cursos y las orientaciones de cursos. En síntesis, pese al sostenido crecimiento de los cursos en línea en la ECE es imperativo trabajar en la constitución de un sistema de aseguramiento de la calidad de los cursos que permita una efectiva apropiación de las TIC para mejorar los procesos de aprendizaje y la formación par la investigación en los estudiantes de las carreras de la ECE.

De igual manera las herramientas de las web 2.0 permitirían a los docentes de la ECE realizar trabajo colaborativo. Por ejemplo, utilizando herramientas como Wiki o Google docs, equipos de docentes de la ECE pueden generar propuestas de investigación, revisiones de literatura, ensayos, artículos y otros tipos de publicaciones sobre campos específicos de la educación que se requieren desarrollar. Asimismo, el software para crear redes sociales puede ser utilizado para el desarrollo de proyectos de investigación. De esta manera, herramientas que permiten interactuar en comunidades, como el foro y la galería de archivos, son muy valiosas para desarrollar los diálogos y el intercambio de información en un equipo de trabajo.

De acuerdo con los planteamientos anteriores, las posibilidades que ofrecen las tecnologías aplicadas a la educación, para mejorar los procesos pedagógicos y favorecer la gestión del conocimiento son diversas y de gran riqueza si se logra el desarrollo de interacciones y relaciones dialógicas de negociación de significados y producción de conocimientos (Garrido, 2003; Rodríguez, 2004; Niemeyer, 2006; 
Lave \& Wenger, 1991). Ello significa reconocer las potencialidades de las TIC pero, sobre todo, reconocer la potencialidad del compartir los pensamientos a través de la palabra, de la discusión y del diálogo en los procesos de producción de conocimientos. Se trata, pues, de recuperar espacios de reflexión y de intercambio entre los académicos de la ECE, sobre los campos del saber que nos ocupa, utilizando las TIC.

No obstante lo anterior, los formadores de los profesionales de la Educación en Costa Rica aún no logran usar intensivamente dichas tecnologías $\mathrm{y}$ no han investigado suficientemente los impactos que la explosión y masificación del uso de las TIC están teniendo en las nuevas generaciones de estudiantes que reciben en sus carreras. Por un lado, los resultados de las investigaciones que se han realizado sobre el impacto de las TIC en las poblaciones aún no se reflejan en los planes de formación de los educadores y la incorporación de las TIC para apoyar los procesos de aprendizaje está en una fase inicial, al menos en los cursos de la ECE. En el campo de la formación para la investigación, al menos en la Cátedra de Investigación Educativa de la ECE, por ejemplo, apenas se están desarrollando pruebas con el uso de comunidades virtuales para apoyar los procesos de diseño y ejecución de proyectos de investigación (Campos y Aguilar, 2011).
A partir del análisis de las condiciones de la ECE, en cuanto a la formación para la investigación, se pueden plantear acciones estratégicas viables y potencialmente efectivas para fortalecer la investigación de manera que sea la actividad clave en el desarrollo de los procesos pedagógicos y de desarrollo de la Escuela. Una de ellas orientada hacia la conformación de comunidades de aprendizaje desde la investigación; una segunda estrategia es la construcción de semilleros de investigación; la tercera consiste en el desarrollo de proyectos y programas de investigación intercátedra y la cuarta estrategia planteada es una efectiva transversalización de la investigación en las asignaturas de los planes de estudio de las carreras de la ECE.

A continuación se describe cada una de esas estrategias propuestas:

La conformación de comunidades de aprendizaje desde la investigación. Consiste en la creación de nuevos espacios y el fortalecimiento de los ya existentes, como las comisiones de autoevaluación y de investigación, para el desarrollo de actividades de análisis y reflexión sobre el accionar de las cátedras y las carreras. También supone la problematización sobre las realidades y la constante identificación de problemas de investigación cuya respuesta permita el mejoramiento de los procesos académicos y el desarrollo profesional del personal docente de la escuela, tal como se observa en la siguiente 


\section{Figura 3}

Comunidades de aprendizaje como estrategia para el fortalecimiento de la generación y la gestión del conocimiento

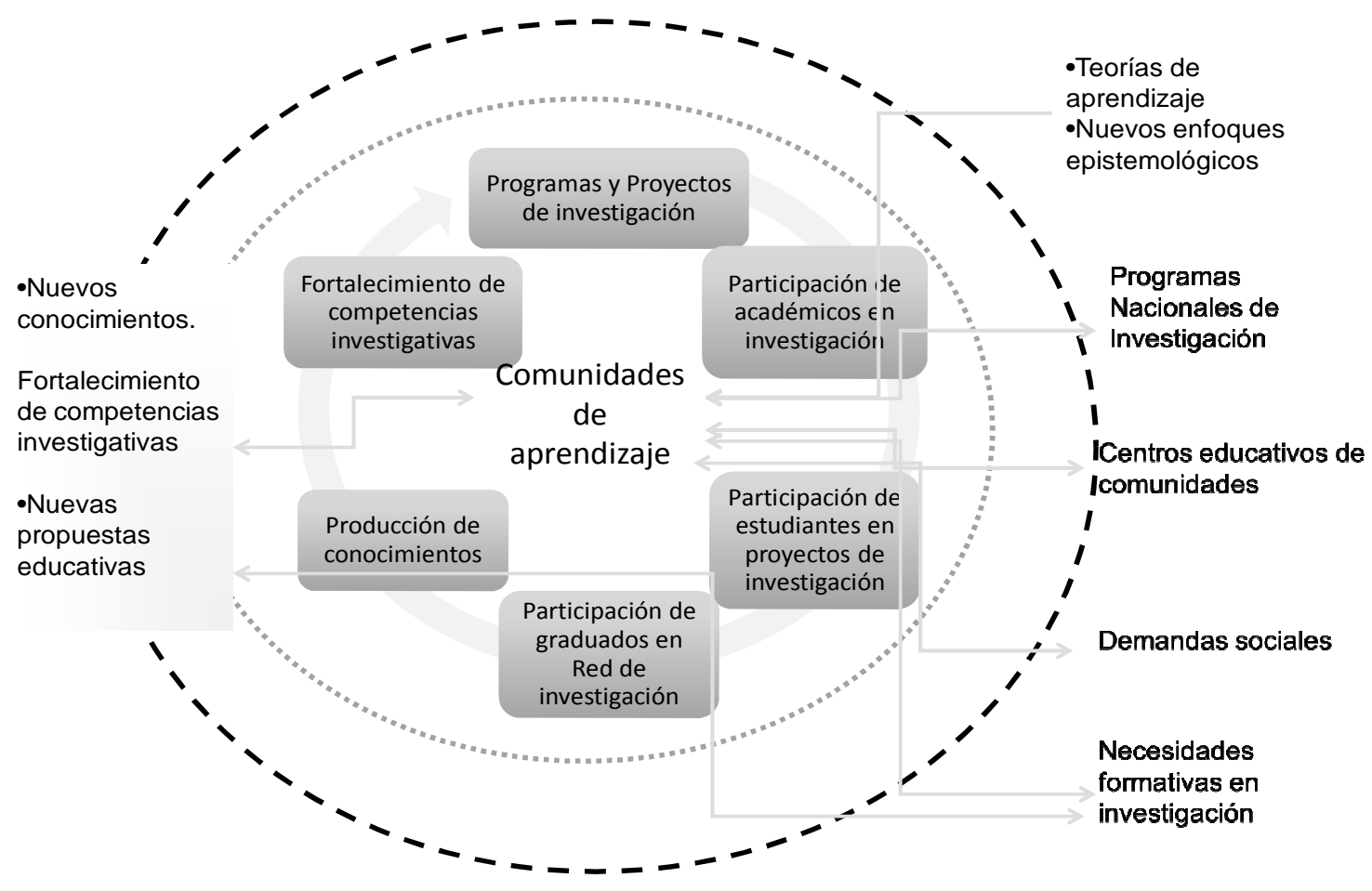

Fuente: Campos, 2010.

El desarrollo de las comunidades Castañeda, González y Oquendo, (2001) denomina grupos o redes temáticas que se pueden potenciar con de aprendizaje puede surgir a partir de experiencias investigativas en proyectos concretos de investigación $\mathrm{o}$ bien pueden constituirse a partir de acciones más específicas, como círculos de estudio sobre temas particulares de interés común, la organización para la sistematización de una experiencia o la construcción de un documento académico, o mediante lo que el uso de herramientas tecnológicas diseñadas específicamente para el desarrollo de comunidades virtuales.

\section{a) Desarrollo de semilleros de investigación \\ Los semilleros de investigación} son estrategias ampliamente utilizadas 
por algunas universidades $\mathrm{y}$, tal como lo plantea Torres (2005), consisten en "espacio de formación, anexa a las labores académicas, y que busca formar investigadores. Es una alternativa y un apoyo para realizar procesos de investigación en que se comparte el conocimiento y la convivencia para ayudar a resolver problemas" (p. 5). Los semilleros de investigación funcionan a partir de la definición y el trabajo sistemático. Bajo algunas líneas de investigación, desarrollan programas de investigación y en estos se inscriben estudiantes y docentes quienes, bajo el acompañamiento de investigadores expertos, logran irse formando y adquiriendo mayor experiencia en el campo investigativo. Progresivamente van constituyéndose como investigadores a la vez que participan en diferentes procesos dentro de los semilleros.

Esta estrategia ha tenido interesantes resultados en universidades latinoamericanas, no solo en cuanto a la producción de conocimientos sobre los campos de estudio en que los programas semilleros se desarrollan, sino también en términos de desarrollo profesional de los docentes participantes y en términos de los procesos formativos de los estudiantes. "Los semilleros de investigación permiten la participación de los estudiantes en la gestión de proyectos de investigación de diferente índole en la que el estudiantes comprende que investigar es un proceso unido a la formación" (Castañeda, et al., 2001 citados por Torres, 2005, p.1).

Experiencias previas han mostrado cómo, a partir de los semilleros de investigación, los participantes logran aprender a plantear problemas, formular hipótesis, desarrollar procesos de recolección de información, diseñar metodologías, trabajar en equipo y discutir los resultados de las investigaciones (Torres, 2005).

\section{b) Proyectos de investigación entre cátedras interdisciplinarios}

Otra estrategia que es, en realidad, lo deseable para el desarrollo de las anteriores estrategias propuestas y que podría ayudar significativamente al desarrollo de la investigación en la escuela, es el trabajo conjunto de diferentes cátedras y programas académicos para investigar sobre un tema específico. El abordaje por parte de académicos de diferentes campos proporciona riqueza en cuanto a la comprensión del fenómeno en estudio, a la vez que se propicia el aprendizaje situado a partir de las realidades de las cátedras en un proceso de aprendizaje colaborativo.

El desarrollo de investigaciones por parte de los grupos de investigación constituidos por encargados de cátedra y de programa es fundamental para expandir la cultura de investigación en la Escuela, para fortalecer las competencias investigativas y para potenciar las cátedras y programas como unidades gestoras y, sobre todo, productoras de conocimiento.

Relacionado con la necesidad de procurar la constitución de grupos de investigación dentro de la Escuela que trabaje sobre las líneas de investigación planteadas por la unidad académica, existe la necesidad de conocer el nivel de dominio competencias investigativas que posee el personal académico, 
especialmente encargados de cátedra y de programa. Para ello es importante promover espacios de autoevaluación de la Escuela que permitan constantemente revisar los avances que se tienen en materia de investigación, no solo el avance en el fortalecimiento de los competencias investigativas sino también en la calidad de la investigación que se desarrolla y el cumplimiento de indicadores mínimos de calidad en cuento a producción científica y el uso de la investigación.

c) Incorporar los resultados de la investigación en las asignaturas de los planes de estudio de la carrera.

Para el caso de la UNED, la investigación forma parte de uno los cuatro ejes curriculares presentes en el quehacer institucional $\mathrm{y}$, pese al importante impulso que se ha logrado luego de la creación de la Vicerrectoría de Investigación en el año 2008, aún hay un largo camino por recorrer hasta que la investigación esté presente en el quehacer de la comunidad académica de la escuela de educación y en el estudiantado.

En este sentido, cobra especial importancia la labor de los encargados de programas, quienes por sus funciones pueden velar por la articulación de las asignaturas que integran el plan de estudios y la efectiva incorporación de la investigación, mediante ejercicios investigativos concretos y debidamente orientados, en las asignaturas y en los Trabajos Finales de Graduación. Para ello, es importante que cada carrera defina sus líneas de investigación en consonancia con las necesidades de desarrollo del campo o campos de conocimiento sobre los que se basa.

Es importante destacar que la cátedra de investigación de la ECE de la UNED, instancia que tradicionalmente ha tenido la principal responsabilidad de formar a los estudiantes en investigación, ha enseñado la investigación primordialmente desde el paradigma positivista con el proceso del "método científico". Por esto, uno de los cambios importantes que deben realizarse, mediante el rediseño de la oferta de cursos, debe ser el replantear la formación en investigación desde otros paradigmas más pertinentes para estudiar la realidad de las aulas escolares (Guba, 1990; Guba \& Lincoln, 1995). Con ello se da un giro en la formación para la investigación desde el fortalecimiento de competencias investigativas y para la investigación como son la capacidad problematizadora, el pensamiento crítico, la capacidad de articular conceptos con el problema y con los hallazgos de la investigación-, más que la enseñanza de los procedimientos de un único método científico.

Por otra parte, en la ECE, la formación para la investigación ha sido restringida al desarrollo de dos cursos de investigación que el estudiante debe llevar en el transcurso de su carrera, lo cual parece ser insuficiente para el logro de competencias investigativas (Moreno, 2005; Restrepo, 2004). De allí que se requiera la construcción de una cultura de investigación y una 
verdadera transversalidad de la investigación en los diferentes cursos de los planes de estudio en los que los estudiantes tengan oportunidad de ir desarrollando competencias investigativas y que los cursos de teoría y metodología de investigación sean un apoyo y no los únicos espacios en los que se ejerciten como futuros docentes investigadores (Day, 2005; Freire, 2004; Stenhouse, 2003).

\section{Conclusiones}

La investigación es una de las funciones académicas de la universidad $y$, en ese sentido, su contribución en el desarrollo social no es solo una decisión interna, sino parte de la misión socialmente conferida a la institución universitaria. En el contexto actual asumir la investigación como una función clave del quehacer para producir, gestionar y utilizar el conocimiento y constituirse en una organización de aprendizaje es una necesidad vital de la academia.

A la luz de lo anterior, la formación de educadores requiere, por un lado, enriquecer los procesos de investigación y, por otro, establecer planes de desarrollo estratégicos orientados al desarrollo basado en la investigación y la gestión del conocimiento. La formación de competencias para la investigación ha de ser una prioridad tanto para los estudiantes como para los formadores y un elemento clave en los perfiles profesionales.

La práctica educativa ha de fundamentarse en la investigación y en sus resultados y debe dar pie a nuevas interrogantes investigativas, de manera que se genere una praxis con la cual se enriquezca el quehacer académico y los procesos de aprendizaje en la formación de docentes. No obstante, ello no acontece sin un previo cuestionamiento de algunas concepciones epistemológicas y de algunos de los elementos básicos sobre los cuales se fundamentan los procesos de enseñanza aprendizaje en la institución educativa. En especial, se trata de repensar los procesos académicos de la ECE para ubicar la producción de conocimiento $\mathrm{y}$ el aprendizaje como el centro; resignificar el quehacer de los encargados de cátedra y de programa dando un lugar clave a su condición de sujetos aprehendientes e investigadores; apostar por lo que Freire denominó una pedagogía de la pregunta más que en la transmisión de las respuestas propuestas por actores externos y, en última instancia, atreverse a producir conocimiento a partir de las realidades que concurren en la ECE.

La calidad educativa y la calidad de la investigación en una carrera de Educación constituyen un tema de vital relevancia. Por ello, los procesos de autoevaluación deben estar imbricados en el quehacer de la academia y, al igual que la investigación, deben constituirse en fuente de conocimiento orientador de procesos de mejora continua de la calidad y del desarrollo.

La formación y el desarrollo de la investigación requieren del diseño de un sistema de gestión de la calidad mediante el cual pueda evaluarse y mejorarse continuamente la labor académica (Salinas, 2001). Tal como indica Bates (2001): "la evaluación para la gestión de la calidad, debe formar 
parte del quehacer diario de las universidades, a efecto de evitar que se convierta en una simple declaración de buenas intenciones... Las buenas intenciones de las universidades no permiten que la pobreza, la desigualdad y la discriminación cedan" (p. 89).

A la luz de lo anterior, es importante que los formadores de formadores asuman la autoevaluación como una práctica habitual en el desarrollo de su labor pedagógica. Según Bolívar (1994), la autoevaluación permite el desarrollo curricular, por lo tanto, las investigaciones que se realicen para alimentar la autoevaluación deben ser desarrolladas por el personal académico de la carrera que se autoevalúa "rompiendo con la tradicional hegemonía del conocimiento externo académico- el propio proceso de desarrollo curricular debe ser concebido como una investigación del profesorado... El profesor reconstruye el currículum cuando, junto a otros compañeros y compañeras, toman propiedad y poder del currículum escolar, generando procesos y formas de trabajo dirigidas a autoevaluar lo que se hace, repensar lo que se podría cambiar y consensuar planes de acción" (p. 15).
En materia de promoción de la investigación para la ECE, es imperiosa la necesidad de establecer una red de investigadores en educación que se involucren en el desarrollo de investigaciones sobre áreas temáticas específicas en el campo de la educación y que, en compañía de miembros del personal académico y de estudiantes (tanto de cursos específicos como de trabajos finales de graduación), logren desarrollo de conocimientos que permitan a la ECE, no solo enriquecer las asignaturas y planes de estudio, sino también incrementar su proyección y el aporte social.

Por otra parte, el monitoreo de los avances en el campo de la educación, específicamente en las líneas de investigación seleccionadas por la ECE, es una necesidad y, para ello, resulta importante que tanto las cátedras como la Comisión de Investigación de la ECE aúnen esfuerzos para establecer un sistema de información que permita mantener al personal académico de la escuela actualizado en los nuevos conocimientos generados en investigaciones en educación $\mathrm{o}$ en campos relacionados.

\section{Referencias}

Aguilar, F. y Campos, J. (2011). Ventana para el intercambio investigativo, comunidad virtual para la formación e Investigación educativa.(en prensa)

Arias, G. E-learning crece en el país. La flexibilidad convierte a la educación en línea en una gran opción para estudiar. Edición 807. El Financiero. Recuperado el 15 de marzo de 2011, de <http://www.elfinancierocr.com/ef_archivo/2011/febrero/27/tecnologia2676523.html> 
Bates. A. W. (2001). Cómo gestionar el cambio tecnológico estrategias para los responsables de centros universitarios. Barcelona, España: Gedisa.

Bolívar, A. (1994). Autoevaluación institucional para la mejora interna. En M. A. Zabalza (1994). Reforma educativa y organización escolar, pp. 915-944. Santiago de Compostela, España: Tórculo. 13

Brunner, J.; Elacqua, G.; Tillet, A.; Bonnefoy J.; González, S. Pacheco, P.; Salazar, F. (2005). Guiar el Mercado. Informe sobre la Educación Superior en Chile. Viña del Mar, Chile: Universidad Adolfo Ibáñez.

Campos, J. y Castro, D. (2010). Docente investigador de su quehacer en Costa Rica. Revista Centroamericana de Educación y Cultura Patria Grande, 1(1).

Campos, J. (2010, octubre). Presentación de propuesta de creación de un centro de investigación ante Consejo de Escuela de Educación. Escuela Ciencias de la Educación. Universidad Estatal a Distancia.

Campos, J. y Chinchilla, A. (2009). Reflexiones acerca de los desafíos en la formación de competencias para la investigación en educación superior. Revista Electrónica Actualidades Investigativas en Educación, 9 (2), pp. 1-20. Recuperado el 20 de agosto del 2010, de <http://redalyc.uaemex.mx/src/inicio/ArtPdfRed.jsp?iCve $=44713058023>$

Campos J. e Hidalgo, R. (2011). La formación para la investigación como eje curricular en la carrera de educación preescolar. (en prensa).

Cañal, P.; Lledo, Á.; Pozuelos, F. y Trave G. (1997) Investigar en la escuela: Elementos para la enseñanza alternativa. Sevilla, España: Díada.

Casas, M. (2005). Nueva universidad ante la sociedad del conocimiento. Revista de Universidad y Sociedad del Conocimiento. 2. (2) 1-18. Recuperado el 22 de abril del 2009, de <http://www.uoc.edu/rusc/2/2/dt/ esp/casas.pdf>

Castañeda, Beatriz; González, Sandra y Oquendo, Sergio. (2001). Semilleros de investigación: una emergencia en pos del conocimiento y la ciudadanía. Medellín, Colombia: Marin Vieco.

Chambeaud, L. y Merlo, P. (s.f). ¿Docentes Investigadores, Docentes Críticos? Una Mirada Desde El Postítulo De Investigación Educativa. Recuperado el 09 de setiembre del 2008, de <http://rapes.unsl.edu.ar/Congresos_realizados/Congresos/IV Encuentro - Oct 2004/eje4/ 30.htm>

Consejo Nacional de Rectores. (2005). Oficina de Planificación de la Educación Superior. Plan Nacional de la Educación Superior Universitaria Estatal 2006-2010.

Crocker, D. Hacia una ética del desarrollo. Revista de Filosofía de la Universidad de Costa Rica. 25 (1987) 129141.

Day, Ch. (2005). Formar docentes: cómo, cuándo y en qué condiciones aprende el profesorado. Madrid, España: Nárcea.

Delors, J. (Coord) (1996). La educación encierra un tesoro. Informe a la UNESCO de la Comisión internacional sobre la educación para el siglo XXI. [En línea] Madrid, España: Santillana. Recuperado el o2 de julio del 2008, de <http://www.bvs.sld.cu/revistas/ems/vol16_1_02/ems04102.htm>

De Miguel, J. (2003). Universidad y Democracia. Cádiz, España: Fundación Municipal de Cultura Ayuntamiento de Cádiz.

De Oliveira, C. (2003). La investigación-acción como estrategia de aprendizaje en la formación inicial del profesorado. Recuperado el 19 de abril del 2009, de <http://www.rieoei.org/rie33ao5.htm>

Díaz, J. (2008). Cambios y Reformas en la Educación Superior. La Educación Superior en América Latina y el Caribe: diez años después de la Conferencia Mundial de 1998. Carlos Tûnnermann B. (Ed.). $\begin{array}{llllll}\text { Recuperado } & \text { el } & 22 & \text { de } & \text { abril } & \text { del }\end{array}$ <http://200.10.229.161/evaluacion/interesantes/Educacion_superior.pdf>

Didriksson, A. (2007). Universidad Sociedad del Conocimiento y Nueva Economía. Recuperado el 19 de abril del 2009, de <http://www.riseu.unam.mx/documentos/acervo_documental/txtidoo16.pdf>

Freire, P. (2004). Pedagogía de la autonomía. Madrid, España: Siglo XXI.

Fromm, C., Ramos, M. y S. V. (2002). La práctica pedagógica cotidiana: Hacia Nuevos Modelos de la Investigación en el Aula. Colección Pedagógica Inicial de Docentes Centroamericanos de Educación Primaria y Básica, pp. 13-23.

Fuentes, S. (2001). La operación ideológica como constitutiva del proceso identificatorio: los educadores ambientales en Mexicali. Recuperado el 18 de abril del 2009, de 


\section{La investigación en la formación de educadores: algunas estrategias para la Escuela de Ciencias de la Educación de la UNED Costa Rica}

http://www.crefal.edu.mx/bibliotecadigital/CEDEAL/acervo_digital/coleccion_crefal/rieda/a20oo_12 3/operacion.pdf

Garrido, A. (2003). El aprendizaje como identidad de participación en la práctica de una comunidad virtual. Recuperado el 24 de abril del 2009, de <http://www.uoc.edu/in3/dt/20088/index.html>

Gibaja, R. (1987). La investigación en Educación. Discusiones y alternativas. Cuadernos $\mathrm{N}^{\mathrm{o}}$ 3. Centro de Investigaciones en Ciencias de la Educación, Buenos Aires, Argentina.

Goulet, D. (1965). Ética del desarrollo. Barcelona, España: Estela-Iepal.

Guba, E. (1990). The paradigm dialogic. [traducción] California, USA: Sage Publications, Inc.

Guba, E. \& Lincoln, Y. (1995). Competing Paradigms in Qualitative Research. [Paradigmas opuestos en investigación cualitativa] en Denzin, N. K. \& Lincoln, Y. Handbook of Qualitative Research. Capítulo 6. California, USA: Sage Publications, Inc.

Hernández, U. (2005). Propuesta curricular para la consolidación de los semilleros de investigación como espacio de formación temprana en investigación. Revista electrónica de la Red de Investigación Educativa. 1 (2). Recuperado el 01 de marzo de 2011, de $<$ http://revista.iered.org/v1n2/pdf/uhernandez.pdf>

Imbernón, F. y Olea, M. (2002). La investigación educativa como herramienta de formación del profesorado: Reflexión y experiencias de investigación educativa. España: Grao.

Kaufman, R., Watkins, R., y Leigh, D. (2001). Useful educational results: Defining, Prioritizing and Accomplishing. Lancaster [traducción]. PA: Proactive Publishing.

Klimovsky, G. (1994). Las desventuras del conocimiento científico. Una introducción a la epistemología. 3 edición, Buenos Aires, Argentina: A-Z Editora

Morales, R. (2011). Aportes educativo, político y social en torno a la investigación con innovación en la UNED Costa Rica. Recuperado el 01 de marzo del 2011, de <http://congreso.inie.ucr.ac.cr/memoria/documentos/1/Aportes\%20educativo,\%20politico\%20y\%20s ocial\%20en\%20torno\%20a\%2ola\%20investigacion-Roxana\%20Morales.pdf>

Moreno, G. (2005). Potenciar la educación. Un curriculum transversal de formación para la investigación. Revista electrónica iberoamericana sobre calidad, eficacia y cambio en Educación. Año 1. España, pp. $520 \quad-540 \quad$ Recuperado el 12 de julio del 2010, de <http://dialnet.unirioja.es/servlet/oaiart?codigo=1130331 (Revista) ISSN 1696->

Muñoz, J. Quintero J. y Munévar, R. (2002). Construcción de teoría a partir de la investigación -acción en el currículo para la formación de educadores. Recuperado el 02 de junio del 2009, de <http://redalyc.uaemex.mx/redalyc/src/inicio/ ArtPdfRed.jsp?iCve=34003405>

Niemeyer, B. (2006, setiembre-diciembre). El aprendizaje situado: una oportunidad para escapar del enfoque del déficit. Revista de Educación, 341, pp. 99-121. Recuperado el 25 de abril del 2009, de <http://www.revistaeducacion.mec.es/ re341/re341_05.pdf>

Ortiz, O. Alexander (2005). Docente - Investigador: ¿Cómo investigar desde el aula de clases?. Recuperado el 15 de abril del 2009, de <http://www.monografias.com/trabajos26/docente-investigador/docentenvestigador.shtml>

Ossa, J. (2005). Educar es enseñar a indagar la investigación como proceso de formación. Recuperado el 19 de abril del 2009, de <http://revistaseletronicas.pucrs.br/ojs/index.php/faced/article/viewFile/430/326>

Programa de Aprendiza en Línea (2010). Informe. Uned

Prensky, M. (2001). Digital Natives, Digital Immigrants.On Horizon. 9 (5). Recuperado el 01 de julio del 2010, de $<>$ http://www.marcprensky.com/writing/default.asp $>$ 
Quintero, J. (2006). Pedagogía universitaria emergente. Revista latinoamericana de Estudios Educativos. 2 (1) $\mathrm{pp} 133-146$

Rama, C. La reingeniería de la educación a distancia en América Latina. Recuperado el 13 de setiembre del 2008, de <http://www.slideshare.net/claudiorama/las-reingenierias-de-las-instituciones-deeducacion-a-distancia-en-america-latina?src=embed $>$

Restrepo, B. (2004). Formación investigativa e investigación formativa: acepciones y operacionalización de esta última. Recuperado el 25 de octubre del 2009, de <http://hermesoft.esap.edu.co/esap/hermesoft/portal/home_1/rec/arc_3529.pdf>

Rodríguez, J. (2007, diciembre). Comunidades virtuales de práctica y de aprendizaje: elementos para una problemática. Revista Electrónica teoría de la Educación y Cultura en la Sociedad de la Información.

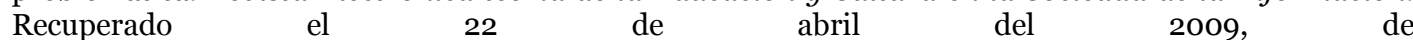
<http://www.publicacions.ub.es/ver_indice.asp?archivo=06969.pdf >

Ruiz, C. (2007). La enseñanza de la investigación en la universidad pública venezolana. Recuperado el 13 de setiembre del 2008, de <http://dialnet.unirioja.es/servlet/articulo?codigo=2310189>

Stenhouse, L. (2003) La investigación y desarrollo del currículum. Madrid, España: Morata.

Torres, Luis Carlos. (2005). Para qué los semilleros de investigación. Recuperado el o1 de marzo de 2011, de <> http://www.revistamemorias.com/edicionesAnteriores/8/semilleros.pdf >

Tünnermann C. y Souza M. (2003). Desafíos de la Universidad en la Sociedad del Conocimiento, Cinco Años Después de la Conferencia Mundial sobre Educación Superior. Artículo producido por el Comité Científico Regional para América Latina y el Caribe del Foro de la UNESCO Paris, diciembre 2003. $\begin{array}{lllllll}\text { Recuperado el } & 25 & \text { de } & \text { abril } & \text { del } & \text { 2009, } & \text { de }\end{array}$ <http://unesdoc.unesco.org/images/oo13/o01344/134422so.pdf>

UNESCO (1998). Declaración Mundial sobre Educación superior en el siglo XXI: visión y acción. Recuperado el 20 de agosto del 2009, de <http://www.unesco.org/ education/educprog/wche/declaration_spa.htm>

Universidad Estatal a Distancia (2004). Modelo Pedagógico. Recuperado el o3 de octubre del 2009, de <http://www.uned.ac.cr/paa/pdf/Materiales-autoev/24.pdf>

Venegas, M. E. (2008). Tendencias de la formación inicial de docentes. Revista Centroamericana de Educación. 3 (1), 271-284. San José, Costa Rica: CECC.

Venegas, M. E. (2009). ¿Cómo se forman los docentes para las escuelas en Centroamérica y República Dominicana. Consolidación de las acciones de mejoramiento de la formación inicial de docentes para la educación primaria en Centroamérica y República Dominicana. San José, Costa Rica: Coordinación Educativa y Cultural Centroamericana.

Vessuri, H. (2009). El rol de la investigación en la educación superior: implicaciones y desafíos para contribuir activamente al desarrollo humano y social. En: La educación Superior en tiempos de cambio. Nuevas dinámicas para la responsabilidad social. Barcelona, España: Global University Network for Innovation.

\title{
Nota acerca del autor
}

\section{Jensy Campos Céspedes}

\author{
Encargada de la Cátedra de Investigación Educativa, en la Universidad Estatal \\ a Distancia (UNED).
}

Correo electrónico: ycampos@uned.ac.cr 